\title{
Bio-inspired Terrestrial Motion of Magnetic Soft Millirobots
}

\author{
Venkatasubramanian Kalpathy Venkiteswaran ${ }^{1}$, Luis Fernando Peña Samaniego ${ }^{1}$, Jakub Sikorski ${ }^{1}$ and Sarthak \\ Misra $^{1,2}$
}

\begin{abstract}
Magnetic soft robots have the combined advantages of contactless actuation, requiring no on-board power source, and having flexible bodies that can adapt to unstructured environments. In this study, four milli-scale soft robots are designed (Inchworm, Turtle, Quadruped and Millipede) and their actuation under external magnetic fields is investigated with the objective of reproducing multi-limbed motion patterns observed in nature. Magnetic properties are incorporated into a silicone polymer by mixing in ferromagnetic microparticles (PrFeB) before curing. The magnet-polymer composite is used to fabricate soft magnetic parts, with pre-determined magnetization profiles achieved using a 1 T field. The resulting soft robots are actuated under external magnetic fields of 10-35 mT which are controlled using an array of six electromagnetic coils. The achieved motion patterns are analyzed over five iterations and the motions are quantified in terms of body lengths traversed per actuation cycle and speed of displacement. The speed of the specimens is calculated to be in the range of $0.15-0.37 \mathrm{~mm} / \mathrm{s}$ for the actuation field used here. The ability of the soft robots to traverse uneven terrain is also tested, with the Turtle and the Millipede demonstrating successful motion.
\end{abstract}

\section{INTRODUCTION}

$\mathbf{N}$ ATURE has always been a source of inspiration to humans, demonstrating a plethora of highly complex but well-optimized systems. Many organisms display a variety of motion capabilities with incredible coordination and control. Much of the research in robot locomotion is centered around biomimetics, to replicate biological motion patterns for adapting to specific environments [1]. Robots have been developed for multi-legged motion on granular media and uneven terrain, flapping wing flight inspired by hummingbirds and insects, and wall-climbing robots have incorporated ideas from geckos [2]-[6]. Drawing inspiration from biological forms is particularly useful in the fields of milli- and microscale robotics, which require efficient functionality and high versatility with a limited amount of material.

Soft robots exhibit greater adaptability to their surroundings compared to rigid-bodied robots, enabling them to deal with dynamic environments, simplifying manipulation tasks such

Manuscript received: September, 10, 2018; Revised January, 5, 2019; Accepted January, 30, 2019.

This paper was recommended for publication by Editor Kyu-Jin Cho upon evaluation of the Associate Editor and Reviewers' comments.

This research has received funding from the European Research Council (ERC) under the European Unions Horizon 2020 Research and Innovation programme (Grant Agreement \#638428 - project ROBOTAR).

${ }^{1}$ Surgical Robotics Laboratory, Department of Biomechanical Engineering, University of Twente, 7500 AE Enschede, The Netherlands. v.kalpathyvenkiteswaran@utwente.nl

${ }^{2}$ Department of Biomedical Engineering, University of Groningen and University Medical Centre Groningen, 9713 GZ Groningen, The Netherlands.

Digital Object Identifier (DOI): see top of this page.

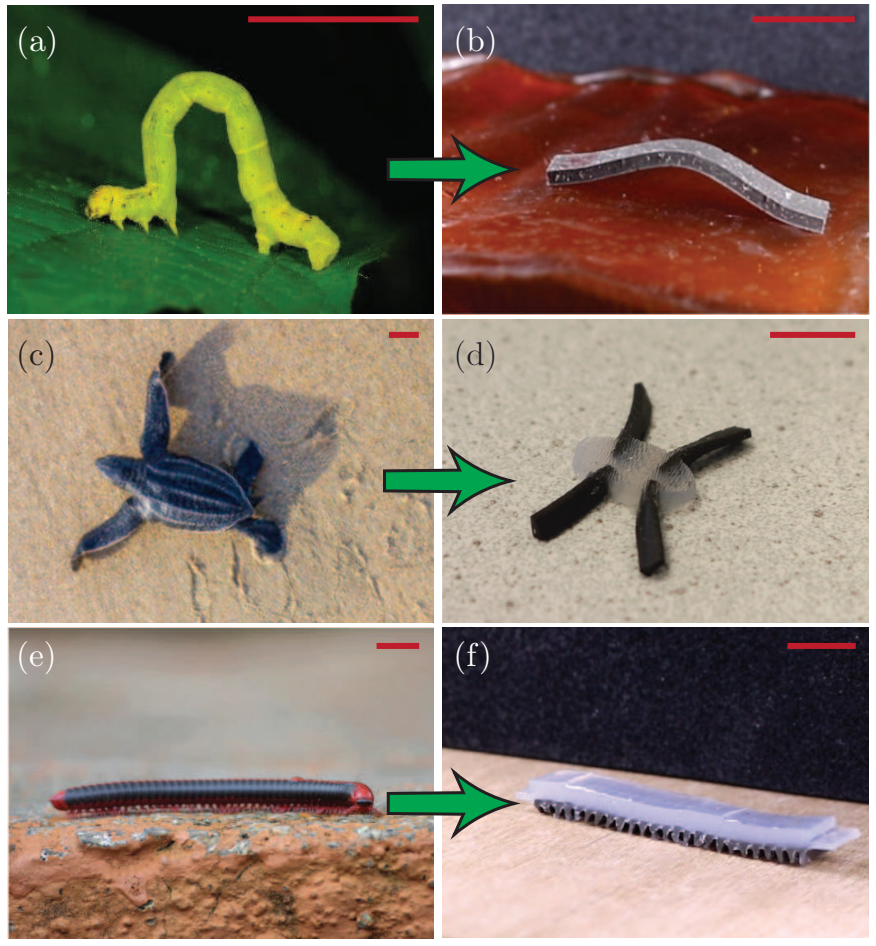

Fig. 1: In this paper, magnetic soft robots capable of bio-inspired terrestrial locomotion are developed. Three examples of biological forms of terrestrial locomotion are shown on the left (C) Getty Images, courtesy of Joe Petersburger, Ben Horton and Naga Sumanth), and the corresponding soft magnetic robots demonstrated in this paper are shown on the right. The first row shows an inchworm (a), whose motion is replicated by a single piece of magnetic polymer (b). The second row shows a turtle moving on land with flippers (c) and its counterpart with four magnetic legs and a non-magnetic body (d). The last row shows a millipede (e), and corresponding soft robot with 25 magnetic legs and a soft non-magnetic body (f). Red scale bar denotes $10 \mathrm{~mm}$.

as grasping and improving mobility over soft substrates [7], [8]. This makes them suitable for applications involving soft biological materials, where their high compliance helps reduce the damage from contact, adapt to different contours in the vasculature, and safely manipulate delicate objects such as organs and cells. Many biological systems also have builtin compliance that is essential for function, and the majority of work in biomimetic soft robots has been on continuumstyle devices, such as worm-like robots exhibiting peristaltic motion and octopus-inspired soft grippers [9], [10]. Actuation for these robots usually involves tendons running along the body of the robot (in the form of shape memory alloys or electric responsive polymers), or pneumatic methods that require channels for fluid flow [11], [12].

Magnetic actuation represents a promising alternative to other actuation methods since it is a non-contact form of energy transfer, which allows for untethered control and can 
be considered as a sterile actuation method for biomedical applications[13]. It is of special interest in the field of medicine because by combining magnetic imaging and actuation, harmful radiation that comes with methods such as fluoroscopy can be eliminated [14] . Magnetic actuation offers rapid response, and as long as the workspace is magnetically transparent, it can be used in different media, including air, vacuum, conducting and non-conducting liquids, and is also unaffected by the ionic concentration of the surrounding medium. Magnetic actuation also eliminates the need for on-board energy storage components, which is favorable when scaling to micro- and nano-scale [15].

For soft robots, magnetic actuation is possible through the combination of magnetic particles with polymers to create responsive materials [16]. In these Magnetic Polymer Composites (MPCs), the magnetic properties depend on the type and morphology of the particles and the volume fraction of particles used; while the mechanical properties are dictated by the polymer matrix [17]. Previously, Garstecki et al. used this technique to develop an elastic swimmer capable of propelling itself in water under the influence of an external rotating magnetic field [18]. $\mathrm{Hu}$ et al. and Diller et al. have demonstrated the use of magnetized soft material to achieve multiple modes of locomotion on solid and fluid interfaces, while Lum et al. presented a method for deriving the necessary magnetization profiles using Fourier series [19][21]. These works used single strip of material, which means the motion is limited when carrying payloads. More recent work has illustrated the use of multiple limbs for grasping (fabricated using 3D printing of ferromagnetic domains), and a multi-legged robot, but with a constant magnetic profile and actuated using permanent magnets, which limits the potential for control[22], [23].

The work presented here describes the design, fabrication and actuation of biomimetic, multi-limbed magnetic soft robots. The primary contribution of this study is the demonstration of multi-limbed motion using patterns previously not utilized in soft robotics, and controlled using electromagnetic coils. The focus is on the reproduction of terrestrial limbdriven motion due to its potential advantages (ability to traverse uneven terrain, carry payload without restricting motion). The motivations for the work are the emerging applications of magnetic composites for untethered systems, primarily in the medical field (such as drug delivery and surgical tool guidance).

\section{BACKGROUND}

The basic concepts behind the motion of the soft robots are detailed in this section. The rationale behind the choice of motion patters is explained first, followed by a brief overview of the theory behind magnetic actuation.

\section{A. Motion patterns}

As described in Sec. I, the focus of this work is on replicating motion patterns in biological organisms that would be suitable for terrestrial locomotion. Multi-limbed locomotion is emphasized, due to the expected benefits of increased

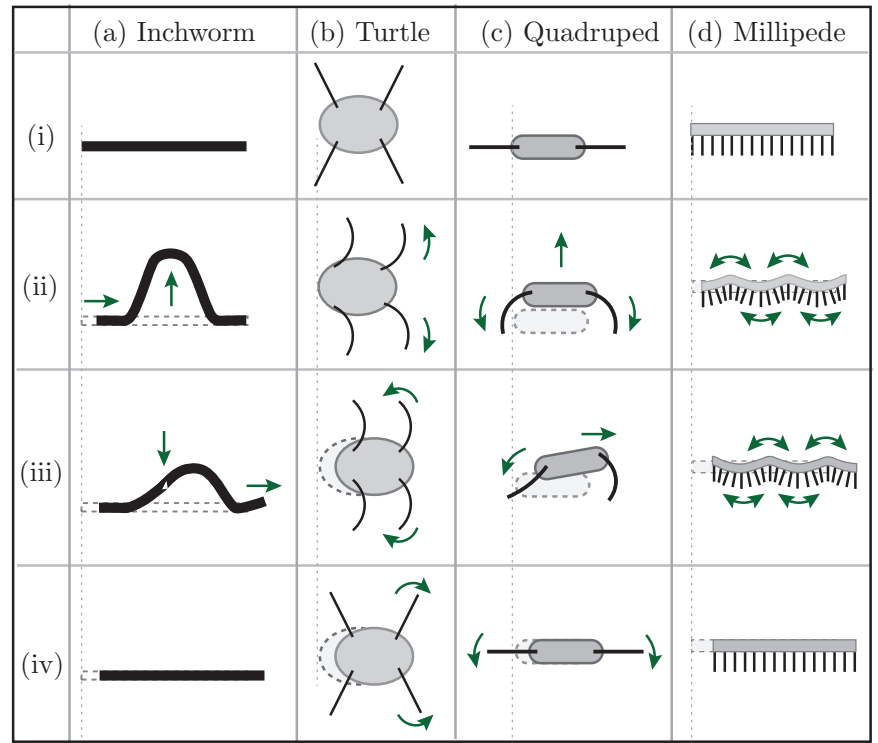

Fig. 2: Schematic representation of four bio-inspired motion patterns demonstrated in this paper. The black segments are made of Magnetic Polymer Composites, whereas the grey parts are elastomer. Each column represents the motion in a single cycle, moving from top to bottom (i-iv). The green arrows represent the motion of the limbs to achieve displacement in each step. All designs are shown from the side, except the Turtle (b) which is shown from above. (a) The Inchworm is a single flat piece of material that moves by anchoring the front end, lifting the center and pulling the rear end forward, followed by anchoring the rear end, relaxing the center and moving the front end forward to return to its flat state. (b) The Turtle has four magnetic flippers attached to a non-magnetic body. The flippers bend to start a rowing motion that generates a reaction force by pushing on the surface (which moves the body forward), and then returns to the initial configuration by lifting the flippers off the surface. (c) The Quadruped also has four legs attached to a non-magnetic body (only two legs are visible in the side view). It follows a similar motion pattern to the inchworm by lifting the body of the surface, leaning forward while anchoring the rear, and relaxing the limbs to return to the flat state. (d) The Millipede has many legs attached to a non-magnetic body, which are subjected to a periodic wave-like displacement profile that propagates along its length, and achieves one cycle of displacement once the wave has passed through all the legs.

stability and potential to overcome uneven terrain. Here, the aim is to demonstrate controllable straight-line motion using varying methods for achieving displacement. Figure 2 shows a schematic representation of the four motion patterns demonstrated in this paper.

One of the most studied biological motions is that of the inchworm (larva of the Geometer moth) [24]. This motion has inspired the development of both soft and hard-bodied robots [25], [26], and is the first pattern studied here (Fig. 2(a)). The inchworm moves by anchoring the front end of its body and dragging the rear end forward, which also involves lifting its central segment. This pattern allows the specimen to be a single continuum and enables motion in either forward or backward direction.

The second motion pattern demonstrated here is inspired by the flipper-driven motion of sea turtles on sandy beaches (Fig. 2(b)). Mazouchova et al. demonstrated the role of twisting of the flipper in achieving this style of motion, and used it to develop a biomimetic robot [27], [28]. Each flipper or limb has a phase in which it makes contact with the ground and generates a reaction force that pushes it forward. In the noncontact phase, the low torsional stiffness allows it to reverse the twist and return to the start of the stroke. This style of motion has multiple points of contact with the surface, which 
enables it to compensate for reduced reaction force from the ground (either from lack of flatness, or due to loose layers, such as in sand).

The third design is also four-legged (named Quadruped, Fig. 2(c)), and is based on the pneumatically-actuated soft robot developed by Shepherd et al. [12]. The motion is similar to that of the inchworm. However, the use of four legs reduces the rise of the central segment and distribution of the legs makes the robot more stable compared to the inchworm.

The final motion pattern illustrated here is that of the centipede or millipede with dozens of limbs. Coordinating each limb individually in this scenario is a challenge, and therefore, these organisms use Central Pattern Generators (CPGs) to achieve locomotion [29]. For straight-line motion, this can be characterized as a metachronal rhythm (sequential action, similar to a periodic wave) of the limbs to generate displacement. Figure 2(d) shows limbs that are repeating the periodic motion of pushing back on the ground and lifting to return to the initial position. This motion is achievable in both directions, requires little lift of body parts, has low risk of instability and is suitable for constrained spaces. In spite of these advantages, there has been limited work on recreating this motion pattern in robotics. The focus in existing work was on generating the motion of each limb rather than the metachronal rhythm that makes this an effective mode of locomotion [30], [31].

\section{B. Magnetic actuation}

The actuation of the soft robots in this work is achieved using external magnetic fields. This is possible through the use of ferromagnetic materials (mixed into an elastomeric polymer). Ferromagnetic materials develop permanent dipole moments $\left(\mathbf{m} \in \mathbb{R}^{3}\right.$ ) when subjected to large magnetic fields. Upon interaction with magnetic fields used for actuation, these dipoles generate mechanical reactions in the forms of forces and torques. The resulting force $\left(\mathbf{F}_{\mathbf{m}} \in \mathbb{R}^{3}\right)$ and torque $\left(\mathbf{T}_{\mathbf{m}} \in \mathbb{R}^{3}\right)$ are calculated using the equations below.

$$
\begin{aligned}
& \mathbf{F}_{\mathbf{m}}=\nabla(\mathbf{m} \cdot \mathbf{B}), \\
& \mathbf{T}_{\mathbf{m}}=\mathbf{m} \times \mathbf{B} .
\end{aligned}
$$

These loads create mechanical stress in the polymer, which leads to deformation. The direction of deformation can be predicted through careful design of the magnetic components, and by controlling the external actuation field as explained in the next section.

\section{MATERIALS AND METHODS}

In this section, the various technical aspects of this work are described. This includes the choice of materials, fabrication techniques, and the experimental setup for actuation of the soft robots.

\section{A. Materials}

The magnetic material used in this work is an isotropic powder made from praseodymium-iron-boron ( $\mathrm{PrFeB})$, with a mean particle size of $5 \mu \mathrm{m}$ (MQFP-16-7-11277, Magnequench
$\mathrm{GmbH}$, Germany). Praseodymium (Pr) is a hard magnetic material (high magnetic remanence), which implies that it is capable of providing strong, permanent magnetic dipole moments $(\mu)$ once magnetized. This property is highly beneficial for magnetic actuation technique used in our robots. A few important properties are listed in Table I.

The polymer into which the magnetic particles are mixed is a silicone rubber (Ecoflex ${ }^{\mathrm{TM}} 00-10$, Smooth-On, Inc., USA). This material also makes up the non-magnetic parts of the soft robots. It has mechanical properties suitable for creating soft and compliant parts, such as low elastic modulus (55.2 $\mathrm{kPa}$ at $100 \%$ strain) and high elongation at break (800\%). The polymer consists of two liquid precursors that need to be mixed and cured to obtain the silicone rubber. The magnetic powder is added before curing in a $1: 1$ ratio by mass to create the MPC.

\section{B. Fabrication}

Figure 3 illustrates the designs of the soft robots. The liquid polymer is degassed in a high vacuum chamber to eliminate air bubbles, and is then allowed to cure at room temperature in molds made from acrylic (Poly-methyl methacrylate). For the Turtle, Quadruped and Millipede, the magnetic parts are made first, and secondary molds are used to add the non-magnetic parts.

After the polymer cures, the magnetic parts are subjected to a field of 1T (B-E 25 electromagnet, Bruker Corp., USA) using fixtures to describe the magnetization profile. The Inchworm is magnetized in a U-shape, with ends perpendicular to the middle segments. For the Turtle and the Quadruped, the legs are magnetized in circular arcs and then attached to the bodies. While the legs for the Turtle are attached such that they bend parallel to the ground plane, for the Quadruped they are attached such that they bend perpendicular to the ground plane. In the case of the Millipede, the legs are separate from each other and cannot be magnetized individually. Therefore, the entire robot is wound on a wooden rod for magnetization such that it makes a helix of 2.5 turns. When straightened, the magnetic profile is a sinusoidal wave of 2.5 cycles (with a tilt of the helix angle, $\alpha$ ). This means that a magnetic field that forces the front end down will lift the back end upwards, which is necessary for straightening or turning maneuvers that will be addressed in future work.

\section{Experimental setup}

The experiments for demonstrating the motion of the soft robots are performed in an array of six electromagnetic coils called BigMag [32]. The coils rotate around a spherical workspace, enabling the system to produce magnetic fields up

TABLE I: Properties of praseodymium-iron-boron (PrFeB) magnetic powder MQFP-16-7-11277 (Magnequench GmbH, Germany).

\begin{tabular}{lr}
\hline Magnetic Property & Value \\
\hline Residual Induction & $960-1000 \mathrm{mT}$ \\
Intrinsic Coercivity & $520-600 \mathrm{kA} / \mathrm{m}$ \\
Coercive Force & $460 \mathrm{kA} / \mathrm{m}$ \\
Magnetization Field (95\% saturation) & $1600 \mathrm{kA} / \mathrm{m}$ \\
Curie Temperature & $345^{\circ} \mathrm{C}$ \\
\hline
\end{tabular}




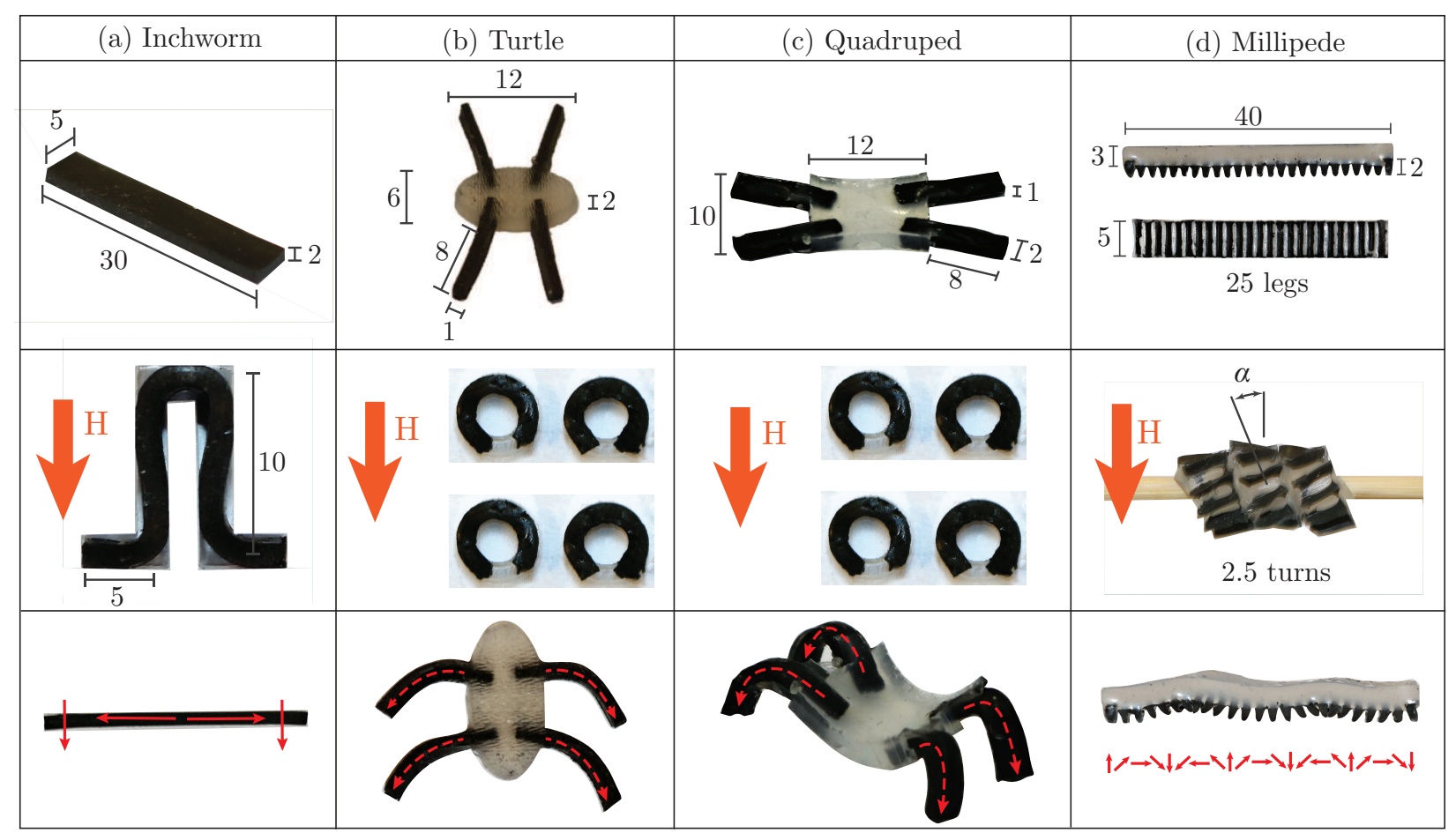

Fig. 3: The four designs demonstrated here are shown with their dimensions in $\mathrm{mm}$ (top row), constrained shapes for magnetization under a large field $(\mathbf{H}=$ $1.0 \mathrm{~T}$ ) (middle row), and the direction of the magnetic dipoles denoted by red arrows (bottom row). Magnetic parts are black and non-magnetic parts are translucent white. (a) The Inchworm is fabricated as a single piece and bent into a U-shape for magnetization, which generates a symmetric magnetic profile. (b) For the Turtle, the legs are magnetized in a circular profile and attached to the non-magnetic body after magnetization such that they bend in the plane of the body. (c) The Quadruped is similar to the Turtle, except that the legs are attached such that they deform perpendicular to the plane of body (to lift it off the ground). (d) The Millipede is made with 25 magnetic legs attached to a non-magnetic body. It is magnetized by winding it in a helix of 2.5 turns on a wooden $\operatorname{rod}(\alpha$ is the helix angle). The magnetization profile of the unwound Millipede is a periodic wave of 2.5 cycles.

to $60 \mathrm{mT}$ in any specified direction with a bandwidth of $40 \mathrm{~Hz}$. The surface for locomotion is a wooden square of $100 \times 100$ $\mathrm{mm}$, fixed at the center of the workspace. Two cameras are used for observing the top and side views of the experiments. A picture of the setup is shown in Fig. 4.

Rotating magnetic fields are used to achieve the desired motion patterns. The fields for each motion pattern are given as functions of time in Table II. The values are selected based on a combination of predicted behavior and observations. Since the focus here is on straight-line motion, most of the fields are in the xz-plane (as defined in Fig. 4), except for the Millipede which requires a tilt equal to the helix angle $(\alpha)$. The time periods $(T)$ were chosen to achieve fastest possible displacement while ensuring the motion patterns are followed accurately. The results of the experiments are presented in the next section.

\section{RESULTS}

The resulting motion of the four designs is illustrated in Fig. 5 , and in the video accompanying the paper. It is noticeable that all the specimens follow the characteristic biomimetic patterns. The actuation cycles are applied repeatedly, and each specimen is capable of reliably moving from one end of the workspace to the other. A few key notes and observations are given below.

For the Inchworm, the magnetic field is asymmetric about the z-axis (as defined in Fig. 4) to produce forward displacement, and can be flipped to reverse the direction of motion. It is easy to fabricate, but is observed to be less stable in the lateral

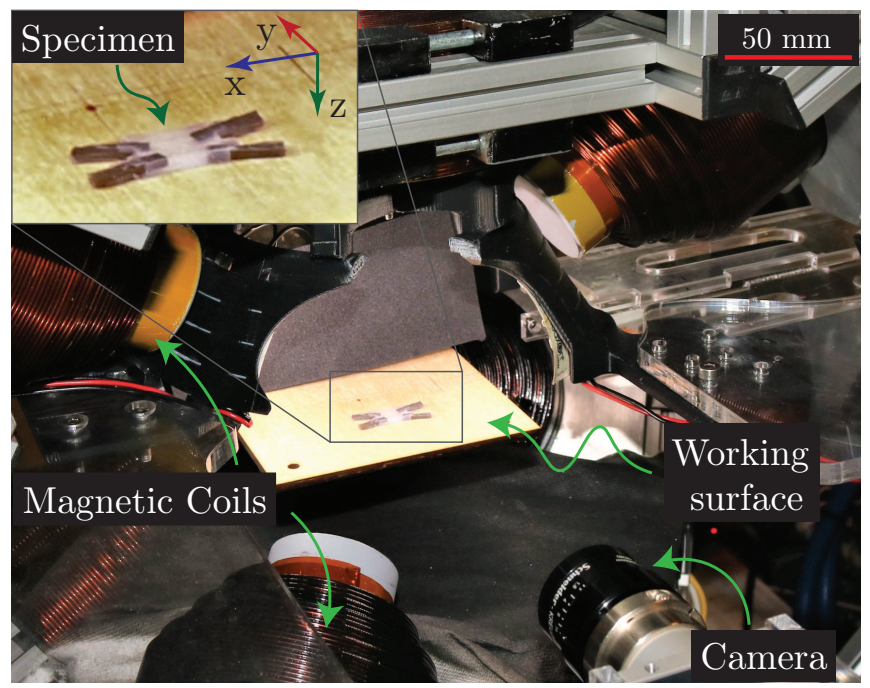

Fig. 4: The setup for magnetic actuation consists of an array of six electromagnetic coils (three coils are visible in the picture). Two cameras provide imaging from the front and top of the workspace. For the experiments in this paper, the working surface is a wooden square of $100 \times 100 \mathrm{~mm}$. The inset shows one specimen on the surface and the coordinate system for defining the magnetic fields.

$y$-direction (falls over to the side). The motion of the Turtle is easily achieved by a rotating field; but the direction cannot be reversed since the magnetic profile of the limbs is biased to one side. It is also highly dependent on the torsion of the limbs, which is evidenced in the images. Increasing the thickness of the limbs by $25 \%$ restricted the motion significantly.

For the Quadruped, achieving forward displacement neces- 
TABLE II: Definitions of magnetic fields for achieving the motion patterns of (a) Inchworm, (b) Turtle, (c) Quadruped and (d) Millipede. The field values $\left(B_{\mathrm{x}}, B_{\mathrm{y}}, B_{\mathrm{z}}\right.$, in $\left.\mathrm{mT}\right)$ are functions of time $(0 \leq t \leq T$, where $T$ is the time period for one cycle), with the directions $(x, y, z)$ defined as shown in Fig. 4. The start and end angles $\left(\theta_{i}\right.$ and $\left.\theta_{f}\right)$ represent the rotation of the magnetic field during a cycle and are measured from the xy-plane.

\begin{tabular}{|c|c|c|c|}
\hline (a) & $\begin{array}{l}B_{\mathrm{x}}(t)=10 \cos \left(\theta_{i}+\left(\theta_{f}-\theta_{i}\right) t / T\right) \\
B_{\mathrm{Z}}(t)=10 \sin \left(\theta_{i}+\left(\theta_{f}-\theta_{i}\right) t / T\right) \\
\end{array}$ & \multicolumn{2}{|c|}{$T=3 s$} \\
\hline (b) & $\begin{array}{l}B_{\mathrm{X}}(t)=12 \cos \left(\theta_{i}+\left(\theta_{f}-\theta_{i}\right) t / T\right) \\
B_{\mathrm{Z}}(t)=12 \sin \left(\theta_{i}+\left(\theta_{f}-\theta_{i}\right) t / T\right)\end{array}$ & $\begin{array}{r}\theta_{i}=0^{\circ} \\
T=\end{array}$ & $\begin{array}{l}\theta_{f}=360^{\circ} \\
=3 s\end{array}$ \\
\hline (c) & $\begin{array}{l}\text { Phase 1 } \\
B_{\mathrm{x}}(t)=15+20 t / T \cos \left(\theta_{i}+\left(\theta_{f}-\right.\right. \\
B_{\mathrm{Z}}(t)=15+20 t / T \sin \left(\theta_{i}+\left(\theta_{f}-\right.\right. \\
\text { Phase 2 } \\
B_{\mathrm{x}}(t)=10 \cos \left(\theta_{i}+\left(\theta_{f}-\theta_{i}\right) t / T\right) \\
B_{\mathrm{Z}}(t)=10 \sin \left(\theta_{i}+\left(\theta_{f}-\theta_{i}\right) t / T\right)\end{array}$ & $\begin{array}{l}t / T) \\
t / T)\end{array}$ & $\begin{array}{c}\theta_{i}=0^{\circ} \\
\theta_{f}=90^{\circ} \\
T=1.5 s \\
\theta_{i}=170^{\circ} \\
\theta_{f}=200^{\circ} \\
T=1.5 s\end{array}$ \\
\hline (d) & $\begin{array}{l}B_{\mathrm{x}}(t)=25 \cos \left(25^{\circ}\right) \cos \left(\theta_{i}+\left(\theta_{f}-\right.\right. \\
B_{\mathrm{y}}(t)=25 \sin \left(25^{\circ}\right) \operatorname{sign}\left(\sin \left(\theta_{i}+(\theta\right.\right. \\
B_{\mathrm{z}}(t)=25 \cos \left(25^{\circ}\right) \sin \left(\theta_{i}+\left(\theta_{f}-\right.\right.\end{array}$ & $\begin{array}{l}t / T) \\
\left.\left.\left.-\theta_{i}\right) t / T\right)\right) \\
t / T)\end{array}$ & $\begin{array}{c}\theta_{i}=0^{\circ} \\
\theta_{f}=360^{\circ} \\
T=1.2 s\end{array}$ \\
\hline
\end{tabular}

sitates splitting the actuation cycle into two phases: one for moving the rear limbs and lifting the body, and another for moving the front limbs forward. For the Millipede, a rotating field is applied in a plane offset from the xz-plane by the helix angle $\left(\alpha=25^{\circ}\right)$. Individual legs move forward and back in a wave-like motion, and lift off the surface (this can be observed more clearly in the video), while the body appears to remain flat. The direction of motion of the Quadruped and the Millipede can be reversed by inverting the direction of rotation of the magnetic field.

The efficacy of the different motion patterns can be compared using the rate of displacement. Table III lists the speed of each specimen using two definitions: displacement per second and body lengths per actuation cycle, along with the deviation in the lateral direction ( $y$-error). The data was collected over five iterations on the motion tests. The Turtle seems most efficient in terms of body lengths per cycle, but its limbs extend laterally, and therefore its length is small compared to its overall size. The average speed of the specimens is in the range of $0.15-0.37 \mathrm{~mm} / \mathrm{s}$, with the Quadruped being the slowest. Naturally, the speed is affected by the time period of the actuation cycle ( shorter cycle $=$ more speed). However, only the Millipede shows reliable performance at low cycle times. This aspect requires further investigation to determine the effects of inertial components and surface interaction on motion generation. The ability of the specimens to follow the same path is illustrated by the mean error in the $y$-direction, which is about $1 \mathrm{~mm}$ for all four soft robots. The displacement with respect to time of each specimen is also presented in Fig. 6 , with the Quadruped having the maximum variation relative to displacement.

The motion of the soft robots is also tested on four non-flat surfaces, as shown in Fig. 7. Surfaces with sharp triangular peaks (a) and smooth sinusoidal profiles (b) are chosen. Each specimen was tested on both surfaces, and also on surfaces with the same profiles inclined at $45^{\circ}$. The Millipede is able to move on the surface (a) (and its inclined version) due to its multiple legs making contact at many points. On the other hand, the Turtle is the only robot capable of motion on surface
TABLE III: Average speed, body lengths (B.L.) covered per actuation cycle and deviation in lateral direction ( $y$-error) of all four specimens over the course of five iterations of the motion experiments.

\begin{tabular}{cccc}
\hline Specimen & Speed $(\mathrm{mm} / \mathrm{s})$ & B. L. per cycle & $y$-error $(\mathrm{mm})$ \\
\hline Inchworm & $0.25 \pm 0.02$ & 0.025 & 0.86 \\
Turtle & $0.35 \pm 0.05$ & 0.087 & 1.21 \\
Quadruped & $0.15 \pm 0.04$ & 0.012 & 0.63 \\
Millipede & $0.37 \pm 0.03$ & 0.011 & 0.95 \\
\hline
\end{tabular}

(b). As seen in Fig. 7 (iv), the Quadruped is able to produce some displacement on the surface profile (b), but is affected by the angle of the peaks and cannot move in a straight line. The Inchworm produces little to no displacement on any of the four tests.

\section{CONCLUSIONS AND FUTURE WORK}

In this paper, magnetic actuation has been used in combination with soft materials to demonstrate bio-inspired terrestrial motion of untethered millirobots. All the designs exhibit controlled and repeatable straight-line motion, while mimicking key characteristics of the biological motion profiles. Specimens with multiple magnetic limbs attached to a nonmagnetic body are demonstrated - a crucial difference from work seen in literature. The motion of the Turtle has previously not been utilized in soft robotics, and has high displacement per actuation cycle. The metachronal rhythm of the Millipede shown here has never been replicated before, and the use of 25 limbs without the need for individual controllers suggests a strong potential for advanced implementation. The multilimbed designs show better performance on non-flat surfaces (compared to the single-piece Inchworm), although improvements must be made to the designs for overcoming different types of obstacles.

The development of small-scale, untethered soft devices could have many applications in areas where remote actuation is necessary. Magnetic actuation also has the advantage of being radiation-free, and does not require power sources on the robots. The work here is restricted to demonstration of straight-line motion, whereas for maneuverability and control, other aspects such as turning, tethering and gripping must be investigated. For surgical applications, miniaturization may be necessary, requiring magnetization to be achieved in-assembly (curing material under magnetic field). Future work will focus on introducing these capabilities into magnetic soft robots and demonstrating specific tasks such as obstacle avoidance and path planning.

\section{REFERENCES}

[1] K.-J. Cho and R. Wood, "Biomimetic Robots," in Springer Handbook of Robotics, B. Siciliano and O. Khatib, Eds. Cham: Springer International Publishing, 2016, pp. 543-574.

[2] U. Saranli, M. Buehler, and D. E. Koditschek, "RHex: A Simple and Highly Mobile Hexapod Robot," The International Journal of Robotics Research, vol. 20, no. 7, pp. 616-631, Jul. 2001.

[3] C. Li, T. Zhang, and D. I. Goldman, "A Terradynamics of Legged Locomotion on Granular Media,' Science, vol. 339, no. 6126, pp. 14081412, Mar. 2013

[4] R. Wood, "The First Takeoff of a Biologically Inspired At-Scale Robotic Insect," IEEE Transactions on Robotics, vol. 24, no. 2, pp. 341-347, 2008. 


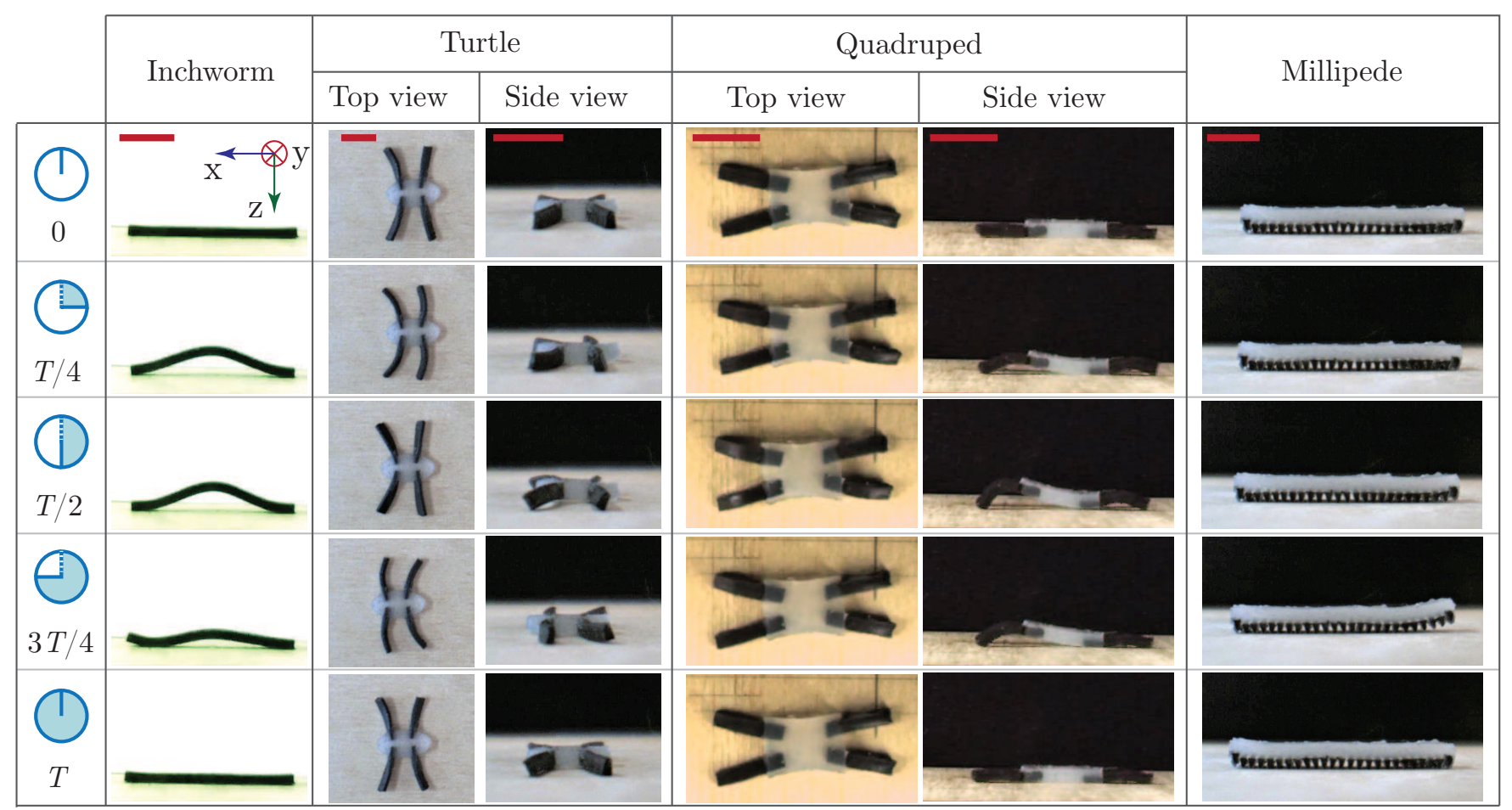

Fig. 5: The resulting motion patterns of the four designs, illustrated using stills from recorded videos. Five frames from a single actuation cycle are shown for each pattern, representing various stages during the cycle (time period $T$ ). The coordinate system used to define the magnetic fields is shown in the top-left image, and the red scale bar denotes $10 \mathrm{~mm}$. See accompanying video for complete motion.
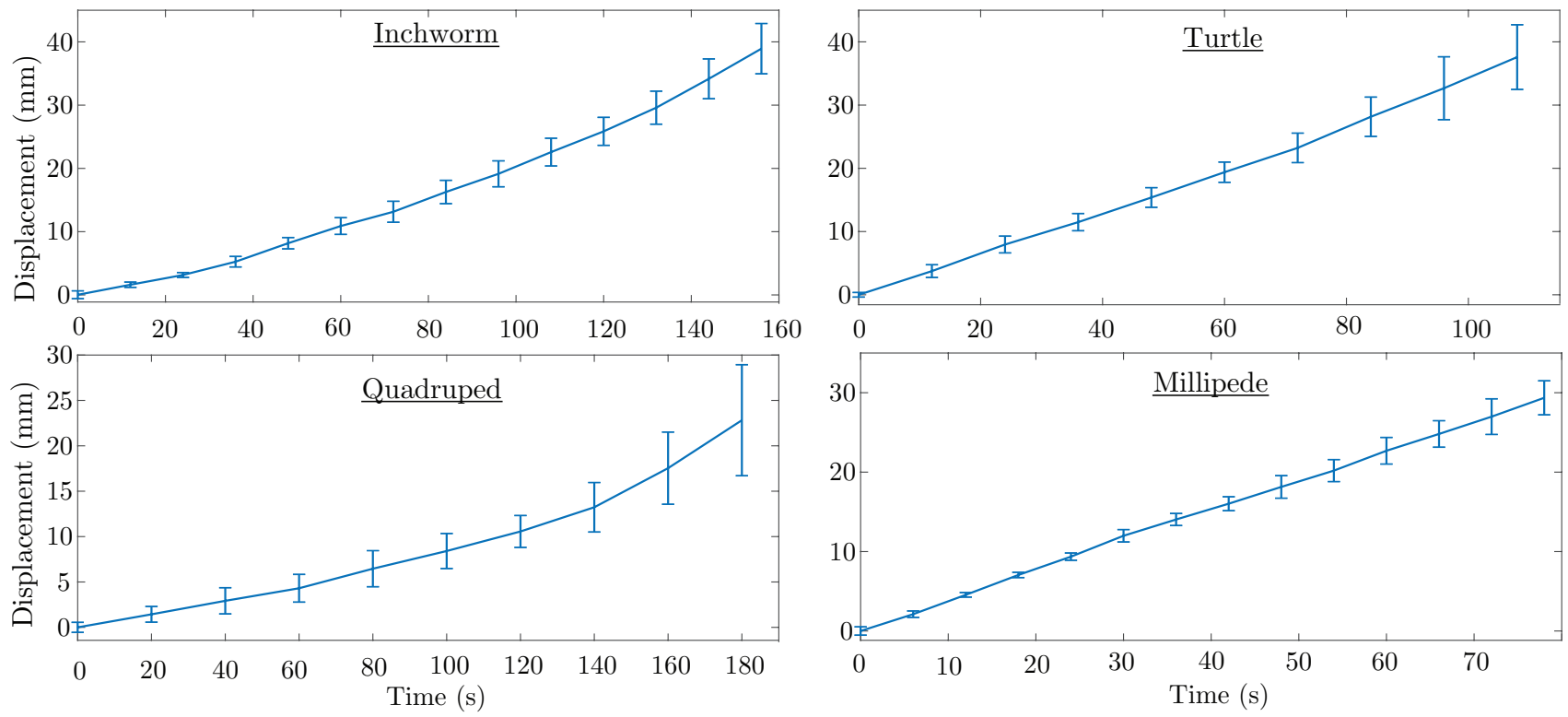

Fig. 6: The displacement of the four soft robots is shown relative to time of motion. The displacement is averaged across five iterations of the tests, and the variation at different points along the path are represented using error bars.

[5] M. Keennon, K. Klingebiel, H. Won, and A. Andriukov, "Development of the Nano Hummingbird: A Tailless Flapping Wing Micro Air Vehicle," in 50th AIAA Aerospace Sciences Meeting including the New Horizons Forum and Aerospace Exposition, Nashville, Tennessee, 2012, p. 588.

[6] S. Kim, M. Spenko, S. Trujillo, B. Heyneman, D. Santos, and M. R Cutkosky, "Smooth Vertical Surface Climbing With Directional Adhesion," IEEE Transactions on Robotics, vol. 24, no. 1, pp. 65-74, Feb. 2008.

[7] S. Kim, C. Laschi, and B. Trimmer, "Soft robotics: a bioinspired evolution in robotics," Trends in biotechnology, vol. 31, no. 5, pp. 287294, 2013.

[8] D. Rus and M. Tolley, "Design, fabrication and control of soft robots," Nature, vol. 521, no. 7553, pp. 467-475, 2015.
[9] S. Seok, C. D. Onal, K. Cho, R. J. Wood, D. Rus, and S. Kim, "Meshworm: A peristaltic soft robot with antagonistic nickel titanium coil actuators," IEEE/ASME Transactions on Mechatronics, vol. 18, no. 5, pp. 1485-1497, Oct 2013.

[10] C. Laschi, M. Cianchetti, B. Mazzolai, L. Margheri, M. Follador, and P. Dario, "Soft Robot Arm Inspired by the Octopus," Advanced Robotics, vol. 26, no. 7, pp. 709-727, Jan. 2012.

[11] H. Lin, G. Leisk, and B. Trimmer, "Soft robots in space: a perspective for soft robotics," Acta Futura, vol. 6, pp. 69-79, 2013.

[12] R. F. Shepherd, F. Ilievski, W. Choi, S. A. Morin, A. A. Stokes, A. D. Mazzeo, X. Chen, M. Wang, and G. M. Whitesides, "Multigait soft robot," Proceedings of the National Academy of Sciences, vol. 108, no. 51, pp. $20400-20403$, Dec. 2011.

[13] J. Kim, S. Chung, S. Choi, H. Lee, and S. Kwon, "Programming 


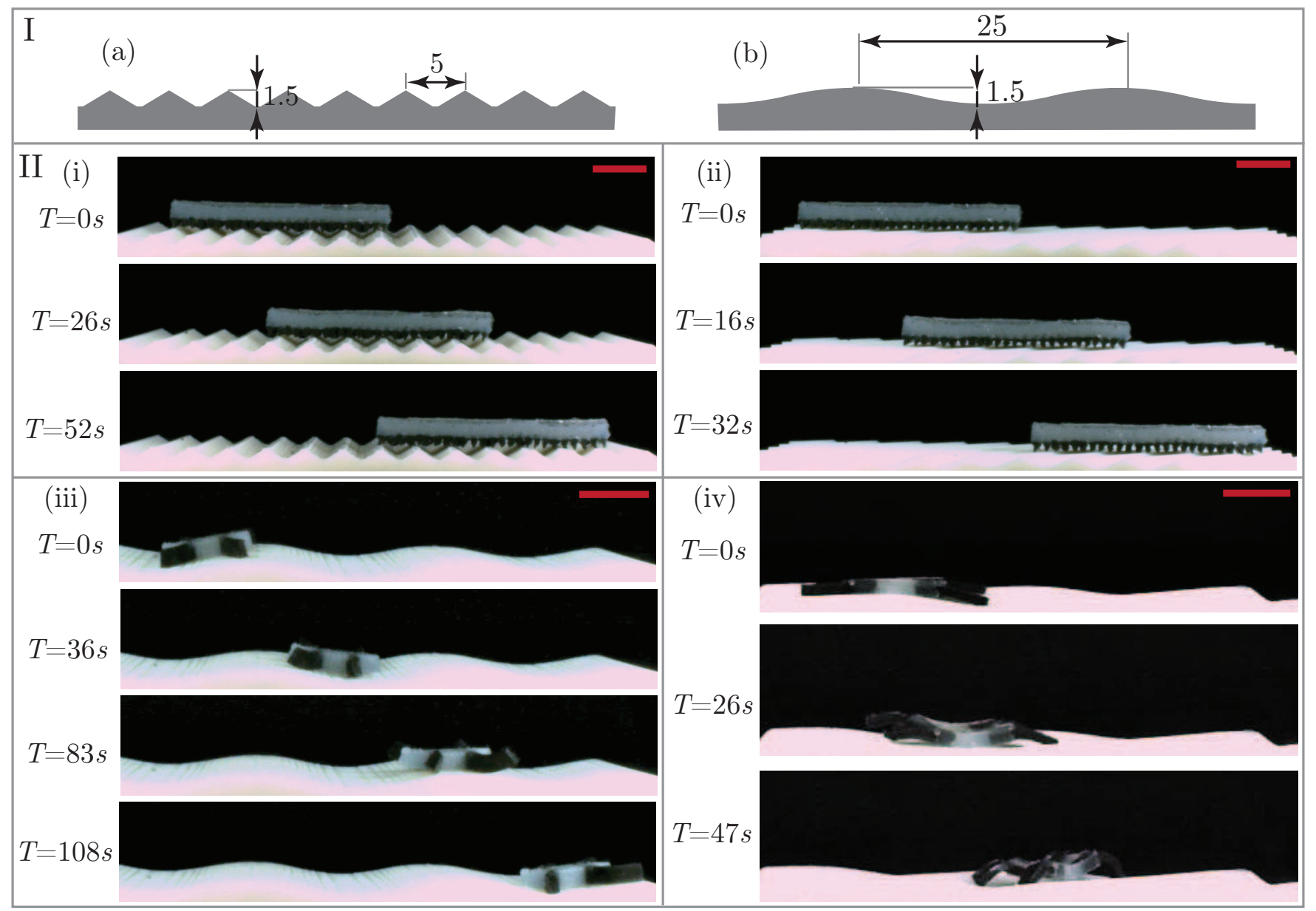

Fig. 7: I. Profiles of two non-flat surfaces: (a) sharp triangular peaks, (b) smooth sinusoidal surface. Dimensions are in mm. II. Results of motion tests on four different surfaces are shown. Tests (i) and (ii) are on surface I(a), while (c) and (d) are on surface I(b). In tests (ii) and (iii), the peaks and troughs are inclined at $45^{\circ}$ to the direction of motion. $T$ is the time of motion and the red scale bar represents $10 \mathrm{~mm}$.

magnetic anisotropy in polymeric microactuators." Nature materials, vol. 10, no. 10, pp. 747-52, 2011.

[14] M. Sitti, H. Ceylan, W. Hu, J. Giltinan, M. Turan, S. Yim, and E. Diller, "Biomedical Applications of Untethered Mobile Milli/Microrobots," Proceedings of the IEEE, vol. 103, no. 2, pp. 205-224, Feb. 2015.

[15] H. Li, T. J. Flynn, J. C. Nation, J. Kershaw, L. S. Stephens, and C. A. Trinkle, "Photopatternable ndfeb polymer micromagnets for microfluidics and microrobotics applications," Journal of Micromechanics and Microengineering, vol. 23, no. 6, 2013.

[16] V. Q. Nguyen, A. S. Ahmed, and R. V. Ramanujan, "Morphing soft magnetic composites," Advanced Materials, vol. 24, no. 30, pp. 40414054, 08/08; 2018/07 2012, doi: 10.1002/adma.201104994; 09.

[17] B. L. Gray, "A review of magnetic composite polymers applied to microfluidic devices," Journal of the Electrochemical Society, vol. 161, no. 2, pp. B3173-B3183, January 012014.

[18] P. Garstecki, P. Tierno, D. B. Weibel, F. Sagues, and G. M. Whitesides, "Propulsion of flexible polymer structures in a rotating magnetic field," Journal of Physics: Condensed Matter, vol. 21, no. 20, 2009.

[19] W. Hu, G. Lum, M. Mastrangeli, and M. Sitti, "Small-scale soft-bodied robot with multimodal locomotion." Nature, vol. 554, no. 7690, pp. 8185, 2018.

[20] E. Diller, J. Zhuang, G. Z. Lum, M. R. Edwards, and M. Sitti, “Continuously distributed magnetization profile for millimeter-scale elastomeric undulatory swimming," Applied Physics Letters, vol. 104, no. 17, p. 174101, 2014.

[21] G. Z. Lum, Z. Ye, X. Dong, H. Marvi, O. Erin, W. Hu, and M. Sitti, "Shape-programmable magnetic soft matter," Proceedings of the National Academy of Sciences, vol. 113, no. 41, pp. E6007-E6015, Oct. 2016.

[22] Y. Kim, H. Yuk, R. Zhao, S. A. Chester, and X. Zhao, "Printing ferromagnetic domains for untethered fast-transforming soft materials," Nature, vol. 558, no. 7709, pp. 274-279, Jun. 2018.

[23] H. Lu, M. Zhang, Y. Yang, Q. Huang, T. Fukuda, Z. Wang, and Y. Shen,
"A bioinspired multilegged soft millirobot that functions in both dry and wet conditions," Nature Communications, vol. 9, no. 1, p. 3944, Sep. 2018.

[24] A. Ghanbari, A. Rostami, S. M. R. S. Noorani, and M. M. S. Fakhrabadi, "Modeling and simulation of inchworm mode locomotion," pp. 617-624, 2008.

[25] K. Kotay and D. Rus, "The inchworm robot: A multi-functional system," Autonomous Robots, vol. 8, no. 1, pp. 53-69, 2000.

[26] W. Wang, J.-Y. Lee, H. Rodrigue, S.-H. Song, W.-S. Chu, and S.-H. Ahn, "Locomotion of inchworm-inspired robot made of smart soft composite (SSC)," Bioinspiration \& Biomimetics, vol. 9, no. 4, p. 046006, 2014.

[27] N. Mazouchova, P. B. Umbanhowar, and D. I. Goldman, "Flipper-driven terrestrial locomotion of a sea turtle-inspired robot," Bioinspiration \& Biomimetics, vol. 8, no. 2, p. 026007, 2013.

[28] N. Mazouchova, N. Gravish, A. Savu, and D. I. Goldman, "Utilization of granular solidification during terrestrial locomotion of hatchling sea turtles," p. 1041, 2010.

[29] M. Golubitsky, I. Stewart, P.-L. Buono, and J. J. Collins, "Symmetry in locomotor central pattern generators and animal gaits," Nature, vol. 401, no. 6754, pp. 693-695, Oct. 1999.

[30] A. Torige, M. Noguchi, and N. Ishizawa, "Centipede type multilegged walking robot," in Proceedings of 1993 IEEE/RSJ International Conference on Intelligent Robots and Systems (IROS '93), vol. 1, Jul. 1993, pp. 567-571 vol.1.

[31] K. L. Hoffman and R. J. Wood, "Myriapod-like ambulation of a segmented microrobot," Autonomous Robots, vol. 31, no. 1, p. 103, May 2011.

[32] J. Sikorski, I. Dawson, A. Denasi, E. E. G. Hekman, and S. Misra, "Introducing BigMag - A novel system for 3d magnetic actuation of flexible surgical manipulators," in 2017 IEEE International Conference on Robotics and Automation (ICRA), May 2017, pp. 3594-3599. 